\title{
Rethinking the Undergraduate Political Science Maj or: The Wahlke Report Revisited
}

Steven Rathgeb Smith, American Political Science Association, smithsr@apsanet.org and

Meghan McConaughey, American Political Science Association, mmcconaughey@apsanet.org

with assistance from Megan Davis, Amanda Grigg, Erin McGrath, and Tanya Schwarz, Betsy Super, American Political Science Association

Presentation at Rethinking the Undergraduate Political Science Major conference

University of North Texas, May 31 - June 2, 2019 


\section{Introduction}

$\triangle$ Review of the Wahlke Report

$\triangle$ Challenges Facing Higher Education

$\triangle$ Data on Trends in Political Science and Social Science Majors

$\triangle$ New Directions for the Major

$\triangleright$ Service Learning and Civic Engagement

$\triangleright$ Conclusion 


\section{The Wahlke Report: \\ Background}

$\triangleright$ Formally "Liberal Learning and the Political Science Major: A Report to the Profession," by John C. Wahlke (1991)

$\triangle$ Report of Task Force on the Political Science Major, appointed by APSA President Lucian Pye

$\triangle$ Part of volume on "Liberal Learning and Arts and Sciences Majors" compiled by Association of American Colleges 


\section{J ohn Wahlke}

$\triangle$ President of APSA (1977-78). He taught at several universities including: Amherst, Vanderbilt, SUNYBuffalo and the University of Arizona.

$\triangle$ He was very active in various APSA related activities related to the undergraduate major including a "syllabus project" in the 1970s. He also chaired a committee on educational policy and programs for the association.

$\triangle$ In the 1960s, he was one of the early Presidents of the Inter-University Consortium for Political Research, now called ICPSR at $U$ of Michigan. 


\section{The Wahl ke Report Recommendations}

\begin{tabular}{|l|l|}
\hline Recommendation 1 & Departments prepare and distribute "Majors' Handbook" \\
\hline Recommendation 2 & $\begin{array}{l}\text { Curriculum include assumptions, methods, and analytical approaches used by } \\
\text { political scientists and by cognate disciplines }\end{array}$ \\
\hline Recommendation 3 & $\begin{array}{l}\text { Departments reexamine their conceptions of "minor field" and "cognate } \\
\text { disciplines" }\end{array}$ \\
\hline Recommendation 4 & Teach most American Government courses in comparative fashion \\
\hline Recommendation 5 & $\begin{array}{l}\text { All relevant courses address ethnic, gender, and cultural diversity and } \\
\text { international and transnational dimensions of particular problems and policies }\end{array}$ \\
\hline Recommendation 6 & $\begin{array}{l}\text { Introductory American Government course emphasize dimensions, and past and } \\
\text { present trends, of ethnic, racial, and cultural diversity in America }\end{array}$ \\
\hline Recommendation 7 & $\begin{array}{l}\text { Departments introduce every political science major to a common set of core } \\
\text { topics }\end{array}$ \\
\hline Recommendation 8 & $\begin{array}{l}\text { Curriculum focus on acquisition of the knowledge and skills to read contemporary } \\
\text { political analyses and development analytic capacity }\end{array}$ \\
\hline
\end{tabular}




\section{New Challenges in Higher}

\section{Education}

$\triangleright$ Unsustainable Costs: how to "bend the cost curve"?

$\triangleright$ Technology innovations and needs: new technologies for education introduce new opportunities but also new demands

$\triangleright$ Balancing skills and humanities instruction in the curriculum: growing demand for new skills such as data science, but need to maintain and make case for humanities education as well 


\section{New Challenges in Higher}

\section{Education}

$\triangleright$ Changing student population: increasing numbers of students of color, low-income students, first generation students, and students at different career and life stages

$\triangleright$ Less-prepared students: increasing number of less-prepared students that need higher levels of support

$\triangleright$ Busier students: increasing number of students with other commitments, particularly work and family obligations 
Challenges Facing Political

Science Discipline and Maj or

$\triangleright$ Trends in enrollments and degrees awarded

$\triangleright$ Distribution of undergrad enrollments

$\triangleright$ Trends in courses and curriculum 
Economics, History, Political Science, and Sociology bachelor's degrees conferred, 1949 - 2016

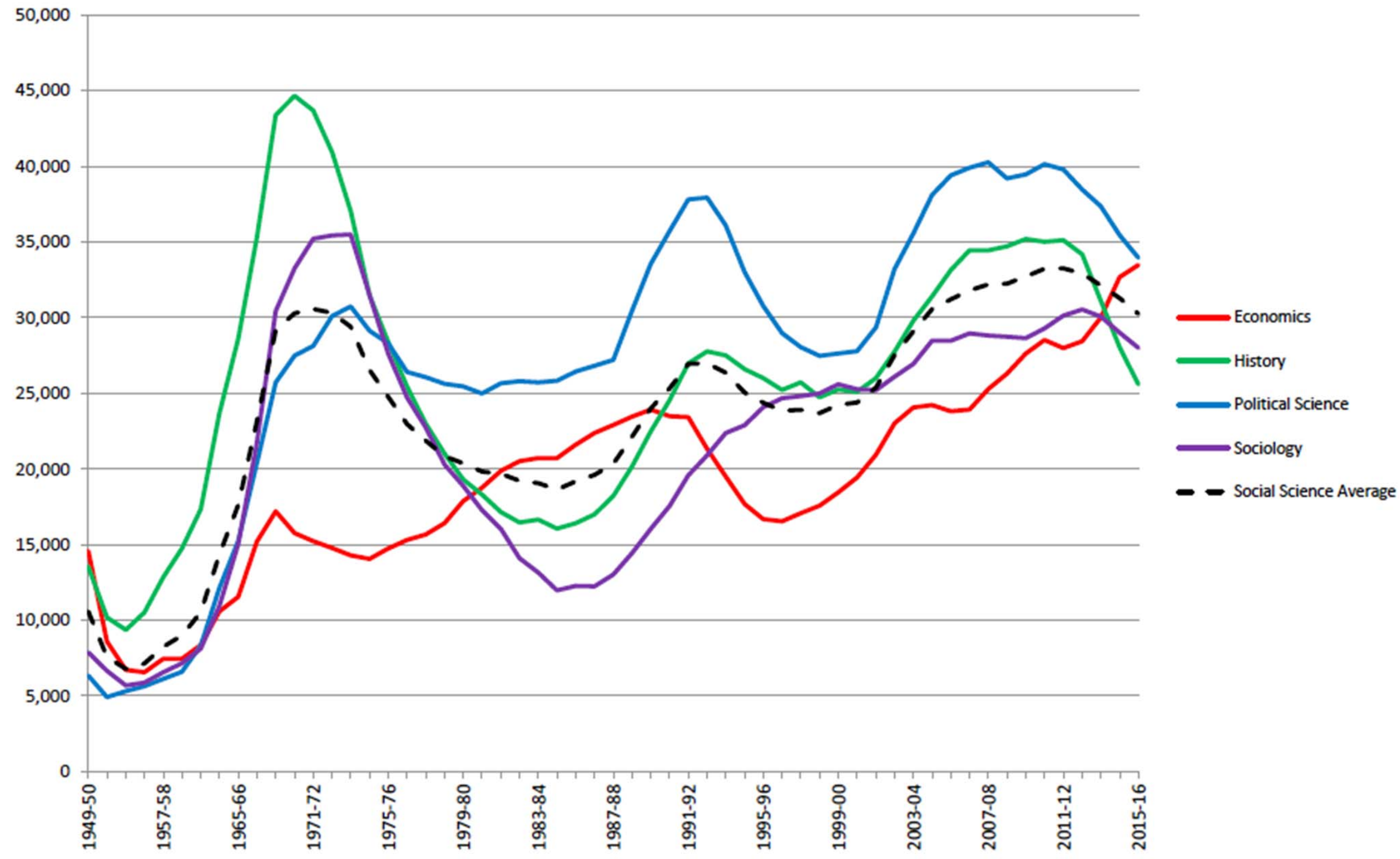

Data from NCES annual Digest of Education Statistics; figure from APSA Trends in Degrees Awarded in the Social Sciences: 1949-2016 Report 
Political Science as a proportion of all Social Science degrees conferred, 1949 - 2016

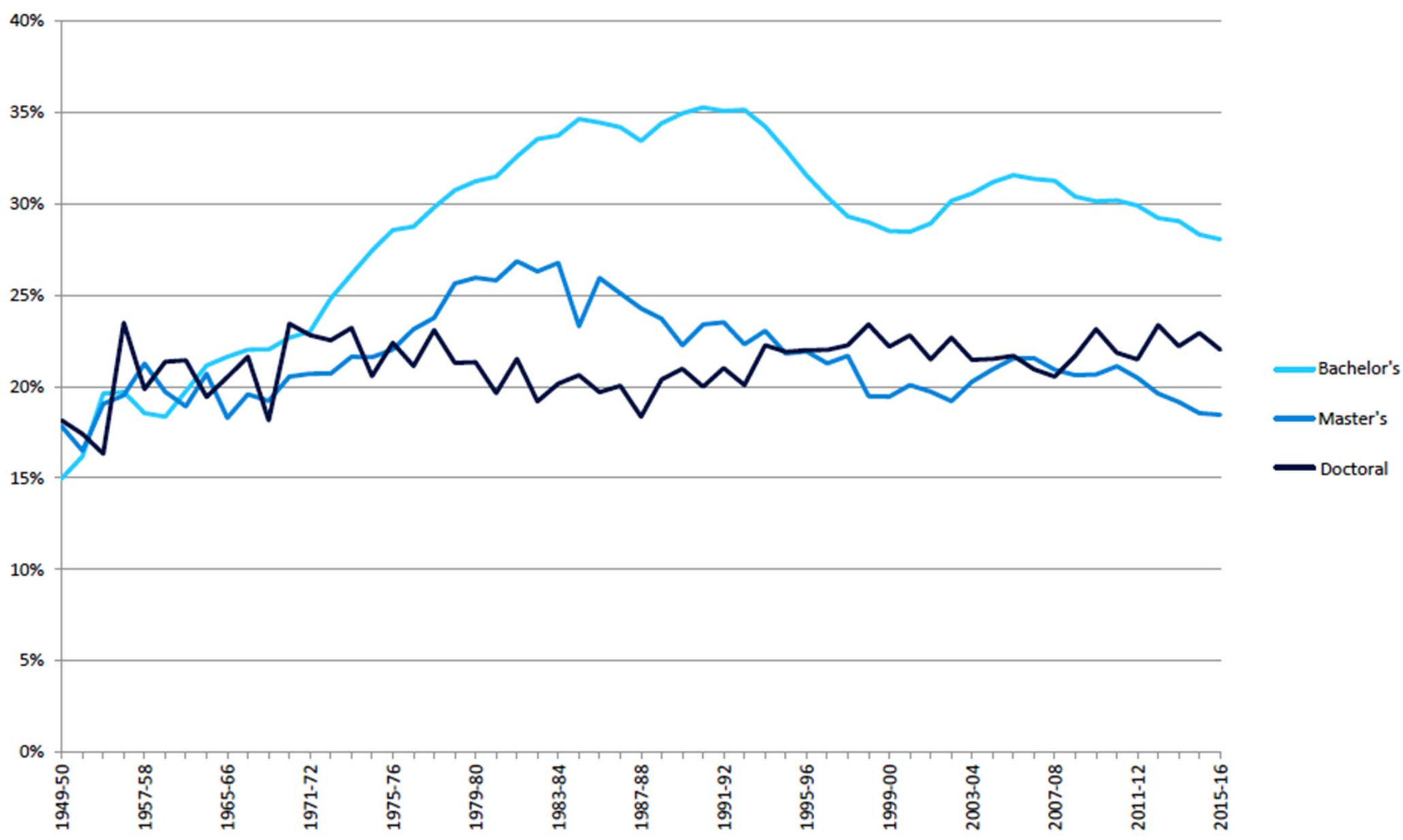

Data from NCES annual Digest of Education Statistics; figure from APSA Trends in Degrees Awarded in the Social Sciences: 1949-2016 Report 


\section{Two-Year Reported Undergraduate Enrollment Changes}

\begin{tabular}{|c|c|c|c|c|c|c|c|c|c|c|}
\hline & \multicolumn{2}{|c|}{$\begin{array}{c}\text { Declined } \\
\text { Significantly }\end{array}$} & \multicolumn{2}{|c|}{ Declined Slightly } & \multicolumn{2}{|c|}{ Stayed the Same } & \multicolumn{2}{|c|}{$\begin{array}{c}\text { Increased } \\
\text { Slightly }\end{array}$} & \multicolumn{2}{|c|}{$\begin{array}{l}\text { Increased } \\
\text { Significantly }\end{array}$} \\
\hline & $\begin{array}{c}2014-15 \\
\text { to } \\
2015-16\end{array}$ & $\begin{array}{c}2016-17 \\
\text { to } \\
2017-18\end{array}$ & $\begin{array}{c}2014-15 \\
\text { to } \\
2015-16\end{array}$ & $\begin{array}{c}2016-17 \\
\text { to } \\
2017-18\end{array}$ & $\begin{array}{c}2014-15 \\
\text { to } \\
2015-16\end{array}$ & $\begin{array}{c}2016-17 \\
\text { to } \\
2017-18\end{array}$ & $\begin{array}{c}2014-15 \\
\text { to } \\
2015-16\end{array}$ & $\begin{array}{c}2016-17 \\
\text { to } \\
2017-18\end{array}$ & $\begin{array}{c}2014-15 \\
\text { to } \\
2015-16\end{array}$ & $\begin{array}{c}2016-17 \\
\text { to } \\
2017-18\end{array}$ \\
\hline All & $2.0 \%$ & $1.9 \%$ & $30.6 \%$ & $23.9 \%$ & $36.7 \%$ & $23.9 \%$ & $25.9 \%$ & $39.0 \%$ & $4.8 \%$ & $11.3 \%$ \\
\hline Public & $2.3 \%$ & $1.1 \%$ & $29.9 \%$ & $20.4 \%$ & $33.3 \%$ & $21.5 \%$ & $27.6 \%$ & $40.9 \%$ & $6.9 \%$ & $16.1 \%$ \\
\hline Private & $1.7 \%$ & $3.0 \%$ & $31.7 \%$ & $28.8 \%$ & $41.7 \%$ & $27.3 \%$ & $23.3 \%$ & $36.4 \%$ & $1.7 \%$ & $4.5 \%$ \\
\hline
\end{tabular}

Data from APSA 2015-2016 and 2017-2018 Departmental Surveys 


\section{New Directions: Workplace}

\section{Skills}

$\triangleright$ Increased focus on workplace-relevant skills: 2016 UCLA Higher Education Research Institute Survey indicates $85 \%$ of first year students across 184 four-year colleges report that "to get a better job" is a "very important" reason they attend college

$\triangleright$ But majors in arts and sciences (arts and humanities, biological sciences, physical sciences, social sciences) report fewer workrelated skills than those in professional fields (education, health, business) (2018 National Survey of Student Engagement ) 


\section{New Directions: Workplace}

\section{Skills}

$\triangleright$ Social science faculty relatively unlikely to "substantially structure courses for job- or work-related knowledge and skills" (just over 50\%)

$\triangleright$ Seniors in arts and humanities, biological, and social sciences, as well as seniors who participated in service learning, more likely to have "unconventional immediate" career plans (internship, travel/gap year, service/volunteering) 


\section{New Directions: Comparative}

\section{Advantages}

$\triangleright$ Workplace Skills:

Social science, communications, education, and social services majors report greater skills in understanding people of other backgrounds

Generally, non-STEM fields more successful in teaching how to work with others and communicate better

How to effectively reframe these as job-related skills

Seniors in social sciences, communications, arts and humanities, and social services report gaining more skills in writing than the average senior

$\triangleright$ Changing Demographics:

$\bigcirc$ Social science, and specifically political science, particularly suited to address changing student demographics by engaging with subjects like race and politics and women in politics 


\section{Role of Service Learning and}

Civic Education

$\triangleright$ Service learning is a key trend in higher education and the social sciences in particular

Nearly $40 \%$ of respondents to 2018 APSA

Community College Faculty survey report that their institution offered internships for credit

$\bigcirc$ New criteria for accreditation from the Higher Learning Commission (effective in 2020) includes specific encouragement for activities that promote informed citizenship and workplace success

$\bigcirc$ A preparedness study by Bentley University indicates that there is demand for civic skills from employers 


\section{Role of Service Learning and}

\section{Civic Education}

$\triangleright$ Service learning and community participation are important for civic education

Matto and Civic Nation argue in Forbes that students must be engaged outside the classroom

$\bigcirc$ Students Learn Students Vote Coalition defines "democratic engagement" to include student

participation in communities and applied learning

$O$ Teaching Civic Engagement Across Disciplines indicates goal is to "impart...skills to peacefully and constructively access [our democratic systems]."

$\triangleright$ Opportunity to connect civic education and workplace skills. Civic engagement education builds skills that can be useful in careers 


\section{Future of the Political Science}

\section{Maj or}

$\triangle$ Restructuring to Emphasize a common core that includes a strong analytics component with strong concentrations and sub-fields.

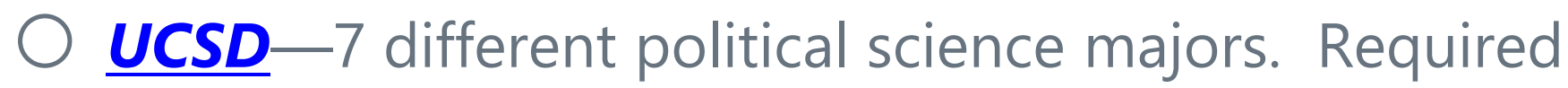
course in Methods and 3 or 4 courses in American Politics, IR, Comparative, and Power and Justice

Stanford-Restructured introductory course which is now called, The Science of Politics. Additional Methods course is required and several courses in one of the 5 tracks: 1) Data Science; 2) Elections, Representation and Governance; 3) IR; 4)Justice and Law; and 5)Political Economy and Development

Duke-Required courses: Data Analysis and one topical courses; choice of courses from sub-fields; other electives; and a capstone

$\bigcirc \underline{\text { UCLA }}-6$ undergraduate political science concentrations, including Race, Ethnicity, and Politics 


\section{Future of the Political Science}

Maj or

$\triangleright$ Restructuring the major to emphasize specific topical areas including civic leadership. (University of Illinois)

$\triangleright$ More in-depth learning and more options for service learning, online instructions, simulations, etc.(San Jose

State) 


\section{Future of the Political Science}

\section{Maj or}

$\triangleright$ Many departments though retain many of the traditional components of the major: required courses in 4 or more sub-fields and several additional more advanced sub-field courses. Capstone experiences and required methods courses remain quite variable.

$\triangleright$ Public Policy undergraduate majors as competitors to political science:

Typical requirements: required stats; ethics; internships; capstone; policy and political analysis

Examples: Duke, USC 


\section{Future of the Political Science}

\section{Maj or}

$\triangle$ Also a growing divide between $\mathrm{PhD}$ and BA institutions, with large PhD granting institutions offering more choice and new curricular pathways in the major.

$\triangleright$ Partially a result of enrollment and hiring trends that allow for more diverse offerings at larger programs

$\triangle$ Partially because larger higher education trends have led to greater contrasts in how different institution types understand higher education and the political science major 


\section{Concluding Thoughts}

$\triangleright$ In many respects, the recent reform efforts are to varying degrees implementing the Wahlke report's recommendations of greater analytic rigor, more structure to the major, and greater responsiveness to student interests and the changing demographics of higher education.

$\triangleright$ Important for these curricular and pedagogical innovations to continue, given the competition for students and greater expectations for accountability for departments and faculty. 
Appendices 
Economics, History, Political Science, and Sociology bachelor's degrees as proportions of all bachelor's uegrees conferred, 1970 - 2016

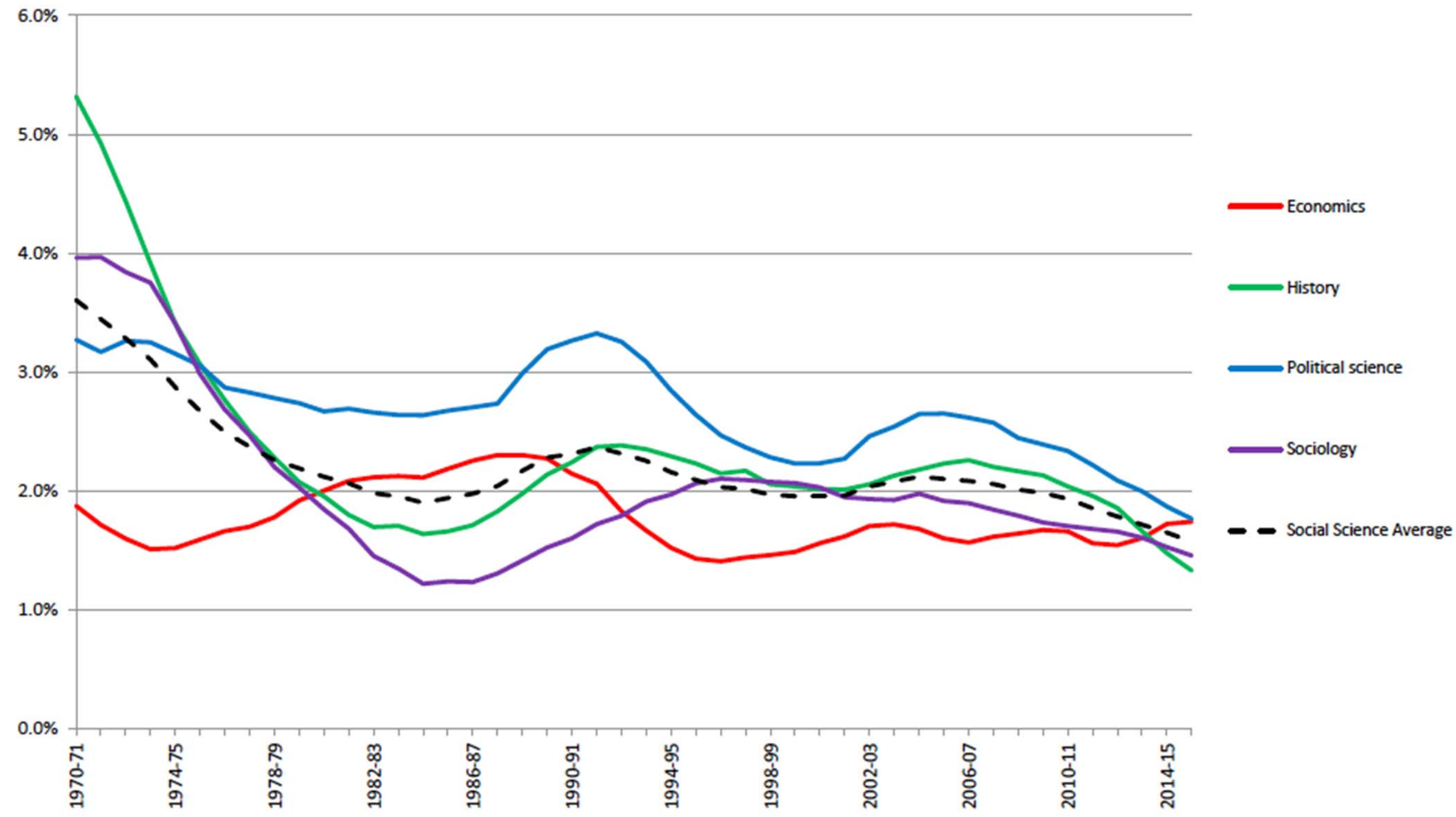

Data from NCES annual Digest of Education Statistics; figure from APSA Trends in Degrees Awarded in the Social Sciences: 1949-2016 Report 


\section{Undergraduate Core-Curriculum Courses by \\ Institution Type and Department Type}

\begin{tabular}{|c|c|c|c|c|c|c|c|}
\hline & & All & PhD & MA & BA & $\begin{array}{c}\text { BA } \\
\text { Combined }\end{array}$ & $\begin{array}{l}\text { Social } \\
\text { Science }\end{array}$ \\
\hline \multirow{3}{*}{$\begin{array}{l}\text { Introduction to } \\
\text { Politics/Political } \\
\text { Science }\end{array}$} & All & $34.3 \%$ & $28.9 \%$ & $37.9 \%$ & $34.2 \%$ & $41.9 \%$ & $29.6 \%$ \\
\hline & Public & $39.0 \%$ & $33.9 \%$ & $41.2 \%$ & $44.1 \%$ & $53.3 \%$ & $23.1 \%$ \\
\hline & Private & $28.8 \%$ & $11.8 \%$ & $14.3 \%$ & $30.0 \%$ & $35.7 \%$ & $35.7 \%$ \\
\hline \multirow{3}{*}{$\begin{array}{l}\text { Introduction to } \\
\text { American Government }\end{array}$} & All & $97.8 \%$ & $98.7 \%$ & $98.3 \%$ & $98.2 \%$ & $97.7 \%$ & $92.6 \%$ \\
\hline & Public & $98.3 \%$ & $98.3 \%$ & $98.0 \%$ & $97.1 \%$ & $100.0 \%$ & $100.0 \%$ \\
\hline & Private & $97.3 \%$ & $100.0 \%$ & $100.0 \%$ & $98.8 \%$ & $96.4 \%$ & $85.7 \%$ \\
\hline \multirow{3}{*}{$\begin{array}{l}\text { Introduction to } \\
\text { International Relations }\end{array}$} & All & $89.9 \%$ & $96.1 \%$ & $94.8 \%$ & $93.9 \%$ & $79.1 \%$ & $63.0 \%$ \\
\hline & Public & $93.0 \%$ & $96.6 \%$ & $94.1 \%$ & $97.1 \%$ & $86.7 \%$ & $61.5 \%$ \\
\hline & Private & $86.3 \%$ & $94.1 \%$ & $100.0 \%$ & $92.5 \%$ & $75.0 \%$ & $57.1 \%$ \\
\hline \multirow{3}{*}{$\begin{array}{l}\text { Introduction to } \\
\text { Comparative } \\
\text { Government }\end{array}$} & All & $79.6 \%$ & $94.7 \%$ & $82.8 \%$ & $78.1 \%$ & $79.1 \%$ & $37.0 \%$ \\
\hline & Public & $84.9 \%$ & $96.6 \%$ & $82.4 \%$ & $85.3 \%$ & $86.7 \%$ & $38.5 \%$ \\
\hline & Private & $73.3 \%$ & $88.2 \%$ & $85.7 \%$ & $75.0 \%$ & $75.0 \%$ & $35.7 \%$ \\
\hline \multirow{3}{*}{$\begin{array}{l}\text { Introduction to } \\
\text { Political } \\
\text { Theory/Ideology }\end{array}$} & All & $73.0 \%$ & $82.9 \%$ & $75.9 \%$ & $73.7 \%$ & $67.4 \%$ & $44.4 \%$ \\
\hline & Public & $76.7 \%$ & $81.4 \%$ & $78.4 \%$ & $73.5 \%$ & $73.3 \%$ & $61.5 \%$ \\
\hline & Private & $68.5 \%$ & $88.2 \%$ & $57.1 \%$ & $73.8 \%$ & $64.3 \%$ & $28.6 \%$ \\
\hline \multirow{3}{*}{$\begin{array}{l}\text { Introduction to } \\
\text { Constitutional } \\
\text { Law/Judicial Politics }\end{array}$} & All & $66.0 \%$ & $63.2 \%$ & $77.6 \%$ & $64.9 \%$ & $58.1 \%$ & $66.7 \%$ \\
\hline & Public & $69.8 \%$ & $66.1 \%$ & $76.5 \%$ & $67.6 \%$ & $60.0 \%$ & $76.9 \%$ \\
\hline & Private & $61.6 \%$ & $52.9 \%$ & $85.7 \%$ & $63.8 \%$ & $57.1 \%$ & $57.1 \%$ \\
\hline \multirow{3}{*}{$\begin{array}{l}\text { Research } \\
\text { Methods/Statistics }\end{array}$} & All & $82.4 \%$ & $94.7 \%$ & $93.1 \%$ & $85.1 \%$ & $67.4 \%$ & $37.0 \%$ \\
\hline & Public & $87.8 \%$ & $93.2 \%$ & $94.1 \%$ & $94.1 \%$ & $73.3 \%$ & $38.5 \%$ \\
\hline & Private & $76.0 \%$ & $100.0 \%$ & $85.7 \%$ & $81.3 \%$ & $64.3 \%$ & $35.7 \%$ \\
\hline
\end{tabular}

Data from APSA 2017-2018 Departmental Survey 


\section{Number of Undergraduate Core Requirements by Institution Type and Department Type}

\begin{tabular}{|c|c|c|c|c|c|c|c|}
\hline & & All & PhD & MA & BA & $\begin{array}{c}\text { BA } \\
\text { Combined }\end{array}$ & $\begin{array}{l}\text { Social } \\
\text { Science }\end{array}$ \\
\hline \multirow{5}{*}{ All } & 1 to 2 & $15.2 \%$ & $26.1 \%$ & $13.6 \%$ & $15.0 \%$ & $4.7 \%$ & $5.6 \%$ \\
\hline & 3 to 4 & $34.4 \%$ & $44.9 \%$ & $37.3 \%$ & $28.3 \%$ & $30.2 \%$ & $33.3 \%$ \\
\hline & 5 to 6 & $30.8 \%$ & $14.5 \%$ & $39.0 \%$ & $32.7 \%$ & $37.2 \%$ & $38.9 \%$ \\
\hline & 7 or more & $18.2 \%$ & $10.1 \%$ & $10.2 \%$ & $23.0 \%$ & $27.9 \%$ & $22.2 \%$ \\
\hline & Don't know & $1.3 \%$ & $4.3 \%$ & $0.0 \%$ & $0.9 \%$ & $0.0 \%$ & $0.0 \%$ \\
\hline \multirow{5}{*}{ Public } & 1 to 2 & $15.2 \%$ & $25.5 \%$ & $15.7 \%$ & $3.0 \%$ & $6.7 \%$ & $9.1 \%$ \\
\hline & 3 to 4 & $40.0 \%$ & $43.6 \%$ & $37.3 \%$ & $33.3 \%$ & $46.7 \%$ & $45.5 \%$ \\
\hline & 5 to 6 & $30.9 \%$ & $18.2 \%$ & $39.2 \%$ & $36.4 \%$ & $40.0 \%$ & $27.3 \%$ \\
\hline & 7 or more & $12.7 \%$ & $9.1 \%$ & $7.8 \%$ & $27.3 \%$ & $6.7 \%$ & $18.2 \%$ \\
\hline & Don't know & $1.2 \%$ & $3.6 \%$ & $0.0 \%$ & $0.0 \%$ & $0.0 \%$ & $0.0 \%$ \\
\hline \multirow{5}{*}{ Private } & 1 to 2 & $15.3 \%$ & $28.6 \%$ & $0.0 \%$ & $20.0 \%$ & $3.6 \%$ & $0.0 \%$ \\
\hline & 3 to 4 & $27.7 \%$ & $50.0 \%$ & $37.5 \%$ & $26.2 \%$ & $21.4 \%$ & $14.3 \%$ \\
\hline & 5 to 6 & $30.7 \%$ & $0.0 \%$ & $37.5 \%$ & $31.2 \%$ & $35.7 \%$ & $57.1 \%$ \\
\hline & 7 or more & $24.8 \%$ & $14.3 \%$ & $25.0 \%$ & $21.2 \%$ & $39.3 \%$ & $28.6 \%$ \\
\hline & Don't know & $1.5 \%$ & $7.1 \%$ & $0.0 \%$ & $1.2 \%$ & $0.0 \%$ & $0.0 \%$ \\
\hline
\end{tabular}

Data from APSA 2017-2018 Departmental Survey 


\section{Undergraduate Enrol Iment Changes from 2016-17 to 2017-18 Academic Year by Institution Type and Department Type}

\begin{tabular}{|c|c|c|c|c|c|c|c|}
\hline & & All & PhD & MA & BA & $\begin{array}{c}\text { BA } \\
\text { Combined }\end{array}$ & $\begin{array}{l}\text { Social } \\
\text { Science }\end{array}$ \\
\hline \multirow{5}{*}{ All } & $\begin{array}{l}\text { Declined } \\
\text { Significantly }\end{array}$ & $3.7 \%$ & $0.0 \%$ & $5.1 \%$ & $4.2 \%$ & $0.0 \%$ & $14.8 \%$ \\
\hline & Declined Slightly & $21.3 \%$ & $19.0 \%$ & $16.9 \%$ & $22.7 \%$ & $25.0 \%$ & $25.9 \%$ \\
\hline & Stayed the Same & $26.2 \%$ & $19.0 \%$ & $16.9 \%$ & $34.5 \%$ & $25.0 \%$ & $33.3 \%$ \\
\hline & Increased Slightly & $38.7 \%$ & $44.3 \%$ & $45.8 \%$ & $31.1 \%$ & $50.0 \%$ & $22.0 \%$ \\
\hline & $\begin{array}{l}\text { Increased } \\
\text { Significantly }\end{array}$ & $10.1 \%$ & $17.7 \%$ & $15.3 \%$ & $7.6 \%$ & $0.0 \%$ & $3.7 \%$ \\
\hline \multirow{5}{*}{ Public } & $\begin{array}{l}\text { Declined } \\
\text { Significantly }\end{array}$ & $2.3 \%$ & $0.0 \%$ & $3.9 \%$ & $2.8 \%$ & $0.0 \%$ & $7.7 \%$ \\
\hline & Declined Slightly & $16.0 \%$ & $18.0 \%$ & $17.6 \%$ & $11.1 \%$ & $7.1 \%$ & $23.1 \%$ \\
\hline & Stayed the Same & $25.7 \%$ & $14.8 \%$ & $19.6 \%$ & $47.2 \%$ & $28.6 \%$ & $38.5 \%$ \\
\hline & Increased Slightly & $41.1 \%$ & $49.2 \%$ & $41.2 \%$ & $25.0 \%$ & $64.3 \%$ & $23.1 \%$ \\
\hline & $\begin{array}{l}\text { Increased } \\
\text { Significantly }\end{array}$ & $14.9 \%$ & $18.0 \%$ & $17.6 \%$ & $13.9 \%$ & $0.0 \%$ & $7.7 \%$ \\
\hline \multirow{5}{*}{ Private } & $\begin{array}{l}\text { Declined } \\
\text { Significantly }\end{array}$ & $5.2 \%$ & $0.0 \%$ & $12.5 \%$ & $4.8 \%$ & $0.0 \%$ & $21.4 \%$ \\
\hline & Declined Slightly & $27.5 \%$ & $22.2 \%$ & $12.5 \%$ & $27.7 \%$ & $33.3 \%$ & $28.6 \%$ \\
\hline & Stayed the Same & $26.8 \%$ & $33.3 \%$ & $0.0 \%$ & $28.9 \%$ & $23.3 \%$ & $28.6 \%$ \\
\hline & Increased Slightly & $35.9 \%$ & $27.8 \%$ & $75.0 \%$ & $33.7 \%$ & $43.3 \%$ & $21.4 \%$ \\
\hline & $\begin{array}{l}\text { Increased } \\
\text { Significantly }\end{array}$ & $4.6 \%$ & $16.7 \%$ & $0.0 \%$ & $4.8 \%$ & $0.0 \%$ & $0.0 \%$ \\
\hline
\end{tabular}

Data from APSA 2017-2018 Departmental Survey 


\section{Undergraduate Enrollment Distributions by Institution Type and Department Type}

\begin{tabular}{|c|c|c|c|c|c|c|}
\hline & & $\mathbf{n}$ & Min & Max & Mean & Median \\
\hline \multirow{6}{*}{ All } & All & 318 & 37 & 15784 & 1784 & 973 \\
\hline & $\mathrm{PhD}$ & 76 & 336 & 15784 & 3889 & 3737 \\
\hline & MA & 58 & 94 & 8390 & 2310 & 1606 \\
\hline & BA & 114 & 37 & 5105 & 869 & 651 \\
\hline & BA Combined & 43 & 53 & 3405 & 612 & 335 \\
\hline & Social Science & 27 & 40 & 4475 & 451 & 205 \\
\hline \multirow{6}{*}{ Public } & All & 172 & 55 & 15784 & 2681 & 1784 \\
\hline & $\mathrm{PhD}$ & 59 & 336 & 15784 & 4452 & 4205 \\
\hline & MA & 51 & 94 & 8390 & 2545 & 1973 \\
\hline & $\mathrm{BA}$ & 34 & 126 & 5105 & 1298 & 1034 \\
\hline & BA Combined & 15 & 125 & 3405 & 958 & 531 \\
\hline & Social Science & 13 & 55 & 4475 & 783 & 335 \\
\hline \multirow{6}{*}{ Private } & All & 146 & 37 & 4803 & 726 & 477 \\
\hline & $\mathrm{PhD}$ & 17 & 548 & 4803 & 1937 & 1735 \\
\hline & MA & 7 & 199 & 860 & 595 & 519 \\
\hline & BA & 80 & 37 & 4611 & 687 & 554 \\
\hline & BA Combined & 28 & 53 & 1932 & 427 & 289 \\
\hline & Social Science & 14 & 40 & 254 & 143 & 146 \\
\hline
\end{tabular}

Data from APSA 2017-2018 Departmental Survey 


\section{Average Undergraduate Enrol Iment in Core Curriculum Courses by Institution Type and Department Type}

\begin{tabular}{|c|c|c|c|c|c|c|c|}
\hline & & All & PhD & MA & BA & $\begin{array}{c}\text { BA } \\
\text { Combined }\end{array}$ & $\begin{array}{l}\text { Social } \\
\text { Science }\end{array}$ \\
\hline \multirow{3}{*}{$\begin{array}{l}\text { Introduction to } \\
\text { Politics/Political Science }\end{array}$} & All & 162 & 245 & 290 & 102 & 80 & 63 \\
\hline & Public & 222 & 265 & 301 & 144 & 114 & 59 \\
\hline & Private & 66 & 50 & 25 & 75 & 53 & 65 \\
\hline \multirow{3}{*}{$\begin{array}{l}\text { Introduction to } \\
\text { American Government }\end{array}$} & All & 495 & 908 & 885 & 178 & 254 & 194 \\
\hline & Public & 821 & 1121 & 990 & 355 & 553 & 328 \\
\hline & Private & 108 & 181 & 138 & 105 & 88 & 48 \\
\hline \multirow{3}{*}{$\begin{array}{l}\text { Introduction to } \\
\text { International Relations }\end{array}$} & All & 169 & 344 & 186 & 90 & 46 & 90 \\
\hline & Public & 237 & 378 & 207 & 141 & 33 & 151 \\
\hline & Private & 82 & 223 & 50 & 68 & 55 & 22 \\
\hline \multirow{3}{*}{$\begin{array}{l}\text { Introduction to } \\
\text { Comparative } \\
\text { Government }\end{array}$} & All & 136 & 264 & 126 & 84 & 45 & 27 \\
\hline & Public & 178 & 286 & 134 & 114 & 36 & 42 \\
\hline & Private & 79 & 180 & 72 & 70 & 51 & 12 \\
\hline \multirow{3}{*}{$\begin{array}{l}\text { Introduction to Political } \\
\text { Theory/Ideology }\end{array}$} & All & 95 & 189 & 87 & 61 & 32 & 25 \\
\hline & Public & 123 & 207 & 93 & 81 & 32 & 31 \\
\hline & Private & 58 & 130 & 26 & 53 & 33 & 14 \\
\hline \multirow{3}{*}{$\begin{array}{l}\text { Introduction to } \\
\text { Constitutional } \\
\text { Law/Judicial Politics }\end{array}$} & All & 60 & 114 & 70 & 41 & 26 & 21 \\
\hline & Public & 68 & 113 & 74 & 63 & 29 & 21 \\
\hline & Private & 38 & 120 & 42 & 31 & 25 & 21 \\
\hline \multirow{3}{*}{$\begin{array}{l}\text { Research } \\
\text { Methods/Statistics }\end{array}$} & All & 82 & 161 & 83 & 44 & 27 & 32 \\
\hline & Public & 110 & 183 & 88 & 57 & 22 & 47 \\
\hline & Private & 44 & 91 & 44 & 38 & 30 & 17 \\
\hline
\end{tabular}

Data from APSA 2017-2018 Departmental Survey 